\title{
The TSC-mTOR Pathway Mediates Translational Activation of TOP mRNAs by Insulin Largely in a Raptor- or Rictor-Independent Manner ${ }^{\nabla}$
}

\author{
Ilona Patursky-Polischuk, ${ }^{1}$ Miri Stolovich-Rain, ${ }^{1} \dagger$ Mirit Hausner-Hanochi, ${ }^{1} \S$ Judith Kasir, ${ }^{1}$ \\ Nadine Cybulski, ${ }^{2}$ Joseph Avruch, ${ }^{3}$ Markus A. Rüegg, ${ }^{2}$ \\ Michael N. Hall, ${ }^{2}$ and Oded Meyuhas ${ }^{1 *}$ \\ Department of Biochemistry, the Hebrew University-Hadassah Medical School, Jerusalem 91120, Israel', Biozentrum, University of \\ Basel, Klingelbergstrasse 70, CH-4056 Basel, Switzerland ${ }^{2}$; and Diabetes Unit, Medical Services, and Department of \\ Molecular Biology, Massachusetts General Hospital and Harvard Medical School, Boston, Massachusetts 02114 ${ }^{3}$
}

Received 22 June 2008/Returned for modification 11 July 2008/Accepted 18 November 2008

\begin{abstract}
The stimulatory effect of insulin on protein synthesis is due to its ability to activate various translation factors. We now show that insulin can increase protein synthesis capacity also by translational activation of TOP mRNAs encoding various components of the translation machinery. This translational activation involves the tuberous sclerosis complex (TSC), as the knockout of TSC1 or TSC2 rescues TOP mRNAs from translational repression in mitotically arrested cells. Similar results were obtained upon overexpression of Rheb, an immediate TSC1-TSC2 target. The role of mTOR, a downstream effector of Rheb, in translational control of TOP mRNAs has been extensively studied, albeit with conflicting results. Even though rapamycin fully blocks mTOR complex 1 (mTORC1) kinase activity, the response of TOP mRNAs to this drug varies from complete resistance to high sensitivity. Here we show that mTOR knockdown blunts the translation efficiency of TOP mRNAs in insulin-treated cells, thus unequivocally establishing a role for mTOR in this mode of regulation. However, knockout of the raptor or rictor gene has only a slight effect on the translation efficiency of these mRNAs, implying that mTOR exerts its effect on TOP mRNAs through a novel pathway with a minor, if any, contribution of the canonical mTOR complexes mTORC1 and mTORC2. This conclusion is further supported by the observation that raptor knockout renders the translation of TOP mRNAs rapamycin hypersensitive.
\end{abstract}

TOP mRNAs are characterized by an oligopyrimidine tract at the $5^{\prime}$ terminus and encode ribosomal proteins, elongation factors, and several other proteins associated with the assembly or function of the translational apparatus (34). The translation of these mRNAs is selectively repressed when proliferation of vertebrate cells is blocked at various phases of the cell cycle by a wide variety of physiological signals and pharmacological treatments or when they are amino acid starved (48). It has previously been shown that translational activation of TOP mRNAs is strictly dependent on the integrity of the phosphatidylinositol 3-kinase (PI3K) pathway $(49,50)$.

Stimulation of PI3K by a variety of growth factor receptors leads to elevated levels of phosphatidylinositol-3,4,5-triphosphate that recruits to the plasma membrane various signaling molecules (reviewed in reference 31). This second messenger lipid participates with 3-phosphoinositide-dependent kinase 1 in phosphorylation (at Thr308) and activation of Akt (also

\footnotetext{
* Corresponding author. Mailing address: Department of Biochemistry, Hebrew University-Hadassah Medical School, P.O. Box 12272, Jerusalem 91120, Israel. Phone: 972-2-6758290. Fax: 972-2-6757379. E-mail:meyuhas@cc.huji.ac.il.

$\S$ Present address: Pediatric Endocrinology Unit, Division of Pediatrics, Chaim Sheba Medical Center, Tel-Hashomer 52621, Israel.

$\dagger$ Present address: Department of Cellular Biochemistry and Human Genetics, The Hebrew University-Hadassah Medical School, Jerusalem 91120, Israel.

$\ddagger$ Supplemental material for this article may be found at http://mcb .asm.org/.

${ }^{\nabla}$ Published ahead of print on 1 December 2008.
}

known as protein kinase B). Akt phosphorylates several cytoplasmic and nuclear substrates and thereby mediates downstream events controlled by PI3K, including cellular and organismal growth, cell survival, and metabolism (31).

The ability of Akt to control cell growth and proliferation has recently been established by the finding that Akt phosphorylates and sequesters the tuberous sclerosis complex 2 (TSC2), an inhibitor of cell growth (see reference 9 and references therein). A heterodimer of this protein with TSC1 operates as a tumor suppressor. Deficiency of either protein is associated with the dominant genetic disorder tuberous sclerosis, characterized by hamartomas with very large cells and in many organs (reviewed in reference 22). The TSC1-TSC2 complex acts as a GTPase activator toward a Ras-like small GTPase, homologous Ras enriched in brain tissue (Rheb), by directly promoting the conversion of Rheb-GTP to Rheb-GDP $(24,53)$ and thus inactivating it. Hence, Akt-induced dissociation of the TSC1-TSC2 complex leads to derepression of Rheb, which then upregulates the mammalian target of rapamycin (mTOR). Rheb binds directly to the catalytic domain of mTOR (30) and activates it by antagonizing mTOR's endogenous inhibitor, FKBP38 (3).

mTOR is a Ser/Thr kinase that controls cell growth, proliferation, and metabolism in response to growth factors, nutrients, and energy stress (reviewed in reference 58). Many of the effects of mTOR are abolished by rapamycin, which exerts its inhibitory effect when complexed with its intracellular receptor, the FK506-binding protein FKBP12 (10). mTOR is involved in two distinct multiprotein complexes. Together with 
raptor, LST8, and FKBP38, it forms the mTOR complex 1 $(\mathrm{mTORC} 1)(3,59)$, which regulates protein synthesis and transcription, as well as autophagy, and displays rapid and ubiquitous FKBP12-rapamycin sensitivity (58). mTORC2, composed of mTOR, rictor, LST8, and SIN1 $(56,59)$, is involved in actin organization and Akt activation (58). mTORC2 phosphorylates Ser473 in the hydrophobic motif of Akt $(21,47)$. TORC2, unlike TORC1, is inhibited by FKBP12-rapamycin only after a prolonged exposure and in a cell type-specific manner (46). The inhibition of mTORC2 by FKBP12-rapamycin is indirect, accounting for the required prolonged incubation time.

Insulin is an important regulator of intermediary metabolism, cell growth, survival, and proliferation. Briefly, binding of insulin to its receptor elicits receptor autophosphorylation, activation, and binding to one of numerous docking proteins, which mediate the various cellular responses. The prevailing dogma suggests that the PI3K-Akt pathway is responsible for most of the effects of insulin on metabolism and cooperates with the Ras-mitogen-activated protein kinase pathway in regulation of cell growth and differentiation (51).

Insulin rapidly regulates protein synthesis by activating the translation factors residing downstream of mTOR, as well as by an Akt-mediated derepression of eukaryotic initiation factor 2 (reviewed in reference 43). However, insulin can also increase protein synthesis capacity by inducing the biosynthesis of the translational apparatus, as exemplified by increased transcription and content of rRNA (2), as well as the synthesis of ribosomal proteins and elongation factor $2(13,28)$.

Here we show that insulin can alleviate the translational repression of TOP mRNAs in serum-starved cells and thus stimulates the accumulation of the protein synthesis machinery. The translational control of TOP mRNAs requires TSC1, TSC2, and Rheb as a deletion of either of the two TSC proteins or overexpression of Rheb renders TOP mRNAs refractory to serum deprivation. mTOR is essential for transduction of the insulin signal to translation efficiency of TOP mRNAs, as its knockdown represses the translation of TOP mRNAs. Surprisingly, this mode of regulation appears to rely on neither mTORC1 nor mTORC2, as rapamycin inhibits the translation of TOP mRNAs only sporadically, and a deficiency of raptor or rictor exerts a marginal effect or no inhibitory effect, respectively, on the translation efficiency of TOP mRNAs. These results suggest that $\mathrm{mTOR}$ exerts its effect on TOP mRNAs via a new pathway that is yet to be determined.

\section{MATERIALS AND METHODS}

Materials used for treating cells. Insulin $(100 \mathrm{nM})$ was from Biological Industries, Kibbutz Beit Haemek, Israel, and LY294002 (50 $\mu \mathrm{M})$, rapamycin (20 nM), FK506 $(20 \mu \mathrm{M})$, aphidicolin $(30 \mu \mathrm{M})$, puromycin $(3 \mu \mathrm{g} / \mathrm{ml})$, and 4-hydroxytamoxifen $(1 \mathrm{mM})$ were from Sigma.

Cell culture. 3T3-L1 (17) preadipocytes were grown as a monolayer in Dulbecco's modified Eagle's medium (DMEM), containing 10\% fetal bovine serum (FBS), in a humidified atmosphere of $7 \% \mathrm{CO}_{2}$ at $37^{\circ} \mathrm{C}$. Differentiation into adipocytes was attained by daily medium replacement until 2 days after the preadipocytes had reached confluence. Two days postconfluence, cells were treated by differentiation medium containing $10 \%$ FBS, $5 \mu \mathrm{g} / \mathrm{ml}$ insulin, $0.5 \mathrm{mM}$ 3-isobutyl-methylxanthine (Sigma), and $0.25 \mu \mathrm{M}$ dexamethasone. Forty-eight hours later, the differentiation medium was replaced with standard medium containing $5 \mu \mathrm{g} / \mathrm{ml}$ insulin for an additional 2 days, and then cells were washed and supplemented with standard medium. At this point, $>95 \%$ of cells acquired the adipocyte phenotype. For study of the responses to insulin stimulation, fully differentiated 3T3-L1 adipocytes were washed and fed with serum-free medium for $48 \mathrm{~h}$ prior to insulin treatment. Human embryonic kidney (HEK) 293 and HEK 293T cells were grown as described previously (20). Transfection of these cells was carried out using the polyethyleneimine procedure. Briefly, $25 \mu \mathrm{l}$ of 2 $\mathrm{mg} / \mathrm{ml}$ polyethyleneimine (average molecular weight, 25,000; Sigma) was added to $750 \mu \mathrm{l}$ of serum-free medium containing $12.5 \mu \mathrm{g}$ DNA. The solution was mixed and kept for $5 \mathrm{~min}$ at room temperature prior to the addition of 60 to $70 \%$ confluent cell culture in a $100-\mathrm{mm}$ plate containing $10 \mathrm{ml}$ complete medium. The medium was changed the next morning, and cells were harvested about $48 \mathrm{~h}$ posttransfection. NIH-IR cells that express the human insulin receptor were grown as previously described (29). Mouse embryonic fibroblast (MEF) lines from $\mathrm{TSC}^{+/+}, \mathrm{p} 53^{-/-}$, and $\mathrm{TSC} 2^{-/-} \mathrm{p} 53^{-/-}$embryos $(60)$ as well as $\mathrm{TSC} 1^{+/+}$ and $\mathrm{TSC}^{-/-}$MEF lines (26) were kindly provided by David Kwiatkowski.

Measurements of cell size and rate of cell division. Measurements of cell size and rate of cell division were performed as previously described (45).

Polysomal fractionation and RNA analysis. Polysomal fractionation and RNA analysis were performed as previously described (50).

Molecular probes. The isolated fragment probes used in the Northern blot analysis were as follows: a 0.97 -kb fragment bearing the $r p L 32$-processed gene, $4 \mathrm{~A}$ (11); a $0.85-\mathrm{kb}$ PCR-generated fragment containing mouse $r p S 6$ coding and flanking sequences (27); a 0.29-kb EcoRI-HindIII fragment containing the mouse $r p S 16$ cDNA (35); a 1.15-kb PstI fragment containing mouse $\alpha$-actin cDNA (36).

Western blot analysis. Immunoblotting was performed as described previously (40) using antibodies against rpS6, phospho rpS6 (Ser235/236 or Ser240/244), phospho S6K1 (Thr389), phospho Akt (Ser473), mTOR, rictor, and raptor (Cell Signaling Technology, Beverly, MA). Exposures were chosen so that the chemiluminescent signals were within the linear response of the film and were quantified by ImageMaster VDS (Amersham Pharmacia Biotech).

Cloning of lentiviral shRNA. Annealed oligonucleotides were cloned into pSuper (7) between the BglII and HindIII sites at the 3' end of the human H1-RNA promoter. A 0.3-kb XhoI-SmaI fragment containing the H1-RNA promoter and the short hairpin RNA (shRNA) coding sequence was subcloned in between the XhoI and EcoRI sites of pSin 18 (18). The sequences of the oligonucleotides are as follows: mTOR shRNA 1 sense, GATCCCCGCCGCATTGTCTCTATCAATTC AAGAGATTGATAGAGACAATGCGGCTTTTTGGAAA; mTOR shRNA 1 antisense, AGCTTTTCCAAAAAGCCGCATTGTCTCTATCAATCTCTTGAA TTGATAGAGACAATGCGGCGGG; Raptor shRNA 1 sense, GATCCCCGGC TAGTCTGTTTCGAAATTTTTCAAGAGAAAATTTCGAAACAGACTAGCC TTTTTGGAAA; and Raptor shRNA 1 antisense, AGCTTTTCCAAAAAGGCT AGTCTGTTTCGAAATTTTCTCTTGAAAAATTTCGAAACAGACTAG CCGGG.

Lentiviral shRNA cloning, production, and infection. pSin 18 coding for shRNA was cotransfected with pCMV $\Delta$ R8 (61) and pMD.G plasmids (37) into HEK 293T cells using DNA-calcium phosphate coprecipitation (20). Viruscontaining supernatant from three $90-\mathrm{mm}$ plates was collected $48 \mathrm{~h}$ after transfection and concentrated by ultracentrifugation for $2 \mathrm{~h}$ at 24,000 rpm in a TST28 rotor at $4^{\circ} \mathrm{C}$. Pellets were resuspended in $400 \mu \mathrm{l}$ supernatant and $30 \mu \mathrm{l}$ of 0.5 $\mathrm{mg} / \mathrm{ml}$ Polybrene (Sigma), $1 \mathrm{ml}$ DMEM was supplemented with $1 \%$ FBS, and this suspension was added to HEK 293 cells in a $60-\mathrm{mm}$ plate. Four hours later, $3.5 \mathrm{ml}$ DMEM supplemented with $10 \%$ FBS was added. Infected cells were serum starved for $48 \mathrm{~h}$ and then 3 -h insulin stimulated on the sixth day. Aliquots of the same cell pellet were used for Western blot and polysomal analyses.

Generation of iRicKO and iRapKO. iRicKO and iRapKO are inducible rictor and raptor, respectively, knockout MEF cell lines (N. Cybulski, M. A. Rüegg, and M. N. Hall, unpublished data). Immortalized MEFs contain a floxed rictor or raptor allele and tamoxifen-inducible Cre recombinase introduced by retroviral infection. Induction of rictor or raptor knockout was accomplished by treating cells with $1 \mathrm{mM}$ 4-hydroxytamoxifen (tamoxifen) for 4 days.

\section{RESULTS}

Insulin induces translational activation of TOP mRNAs by stimulating cell division or cell growth. Previous studies have shown that insulin-stimulated synthesis of ribosomal proteins and elongation factor 2 in resting vertebrate cells is resistant to actinomycin D $(13,28)$. These results indicated that insulin exerts its effect posttranscriptionally, yet the precise mechanism, translational activation, or stabilization of the protein product has not been resolved. Therefore, we set out to explore the effect of the hormone on the translation of TOP mRNAs. 
A

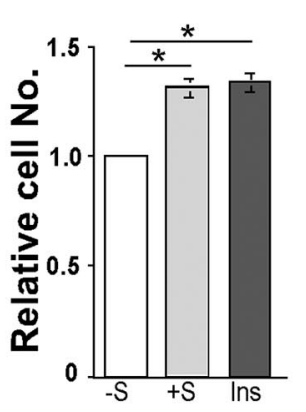

D

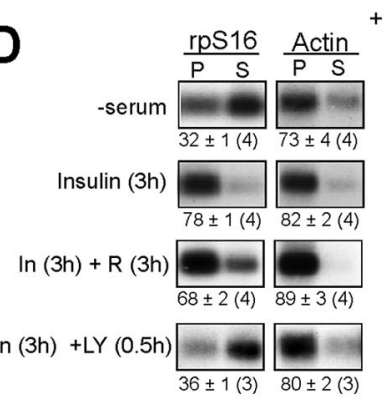

B

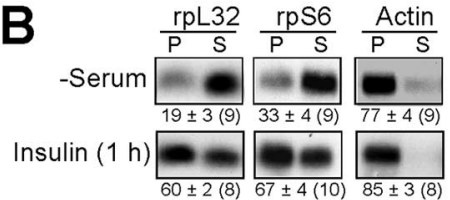

$+\mathrm{LY}(0.5 \mathrm{~h})$

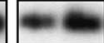
$28 \pm 2(3)$

+Rapa (1

Insulin (3 h)
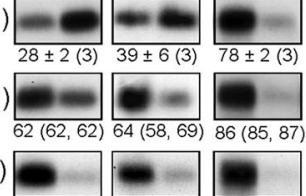

Rapa (3 h)
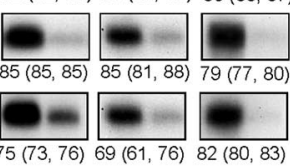

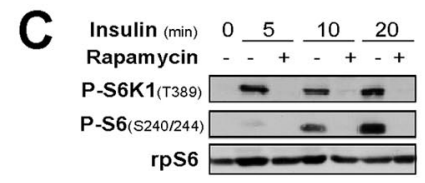

$E$

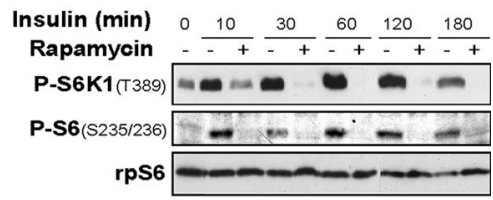

$\mathbf{F}$

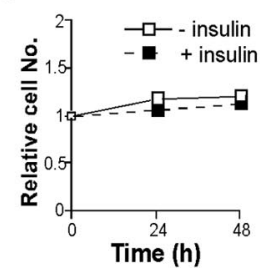

G

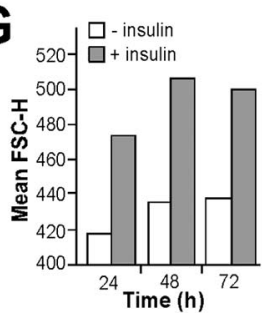

H

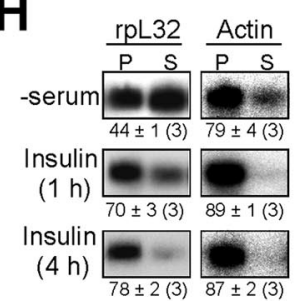

FIG. 1. Insulin induces translational activation of TOP mRNAs. (A) NIH-IR cells were serum-deprived for $72 \mathrm{~h}$ and then kept starved ( $-\mathrm{S})$, serum refed $(+\mathrm{S})$, or insulin-treated (Ins). Twenty-four hours later, the cell number was assessed by the methylene blue staining protocol (39). The number of cells following each treatment was normalized to that of serum-starved cells, which was arbitrarily set at 1 and is presented as the mean \pm the standard error of the mean (SEM; $n \geq 10$ ). *, $P<0.05$. (B) NIH-IR cells were serum starved for $72 \mathrm{~h}$ and then insulin treated in the absence or presence (+Rapa) of rapamycin for the indicated time or LY294002 for the last $0.5 \mathrm{~h}(+\mathrm{LY}$ [0.5 h]). Subsequently, cells were harvested and cytoplasmic extracts were prepared. These extracts were centrifuged through sucrose gradients and separated into polysomal (P) and subpolysomal (S) fractions. RNA from equivalent aliquots of these fractions was analyzed by Northern blot hybridization with cDNAs corresponding to TOP mRNAs (encoding rpL32 and rpS6) and a non-TOP mRNA (Actin). The radioactive signals were quantified, and the relative translational efficiency of each mRNA is numerically presented beneath the autoradiograms as a percentage of the mRNA engaged in polysomes. These figures are expressed as an average \pm SEM of the number of determinations in parentheses or the average with the individual values in parentheses if only two determinations are presented. (C) NIH-IR cells were serum starved for $72 \mathrm{~h}(0)$ and then either insulin treated without $(-)$ or with $(+)$ rapamycin for the indicated amount of time. The cytoplasmic proteins were subjected to Western blot analysis using the indicated antibodies. (D) HEK 293 cells were serum starved for $48 \mathrm{~h}$ and insulin treated for the last $3 \mathrm{~h}$ without or with rapamycin (R) or with LY294002 (LY) for the last $0.5 \mathrm{~h}$. Cells were harvested and subjected to polysomal analysis. (E) HEK 293 cells were serum starved for $48 \mathrm{~h}(0)$ and then treated with $100 \mathrm{nM}$ insulin without $(-)$ or with $(+)$ rapamycin for the indicated amount of time. The cytoplasmic proteins were subjected to Western blot analysis. (F) Confluent cultures of 3T3-L1 preadipocytes were induced to differentiate, and when more than $90 \%$ of the cells converted into adipocytes, they were serum starved for $48 \mathrm{~h}$ and then kept starved with or without insulin for up to $48 \mathrm{~h}$. At the indicated time, cells were trypsinized and counted. Numbers of cells (average of at least three repetitions for each time point) were normalized to the number at time zero. (G) Adipocytes were serum starved for $48 \mathrm{~h}$ and then kept starved (-insulin) or treated with insulin for the indicated amount of time. The size of cells was determined as described in "Materials and Methods" and is presented as FSC-H. (H) Adipocytes were serum starved for $48 \mathrm{~h}$ and then harvested or insulin treated for 1 or $4 \mathrm{~h}$. Subsequently, cells were harvested and subjected to polysomal analysis.

Initially, we showed that proliferation of serum-starved mouse fibroblasts (NIH-IR) could be induced by insulin as efficiently as could be achieved by serum replenishment (Fig. 1A). Accordingly, TOP mRNAs encoding rpL32 and rpS6 were rapidly (within $1 \mathrm{~h}$ ) recruited into polysomes upon insulin treatment (Fig. 1B). This translational activation was blocked when insulin-treated cells were exposed to the PI3K inhibitor LY294002 for $0.5 \mathrm{~h}$. In contrast, rapamycin treatment for $1 \mathrm{~h}$ or even $3 \mathrm{~h}$ had little to no effect in both NIH-IR and HEK 293 cells (Fig. 1B and D), even though the drug fully inhibited mTORC1 within $10 \mathrm{~min}$, as monitored by the phosphorylation status of the mTORC1 targets S6K1 and rpS6 (Fig. 1C and E).

We have previously shown that the translation of TOP mRNAs can be activated in resting PC12 cells when induced to grow in size by nerve growth factor (49). Here we show that insulin failed to induce proliferation of terminally differentiated adipocytes (Fig. 1F) but was able to induce their growth
(Fig. 1G), including the translational activation of rpL32 (Fig. 1H). It appears, therefore, that insulin can induce translational activation of TOP mRNAs by eliciting cell growth in the presence or absence of cell division.

The translation of TOP mRNAs is resistant to mitotic arrest in cells deficient for TSC1 or TSC2. Translational activation of TOP mRNAs by serum is fully reliant on the integrity of the PI3K-Akt pathway $(49,50)$. We reasoned, therefore, that Akt might exert its stimulatory effect on TOP mRNAs via phosphorylation and inactivation of the tumor suppressor TSC1TSC2 complex. This complex is active in serum-starved cells, and therefore it is conceivable that the translation of TOP mRNAs might be resistant to serum starvation in cells deficient for either TSC1 or TSC2. Indeed, polysomal analysis demonstrated that $\mathrm{rpL} 32$ mRNA remained efficiently translated (mostly in polysomes) in $\mathrm{TSC}_{2}^{-/-} \mathrm{MEFs}$, but not in $\mathrm{TSC}^{+/+}$ MEFs, when arrested either in $G_{0}$ by serum starvation or in $S$ 
A
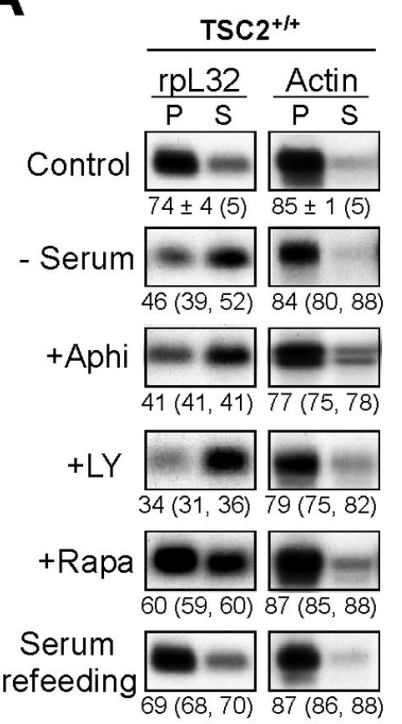

$\mathrm{TSC}^{-/-}$
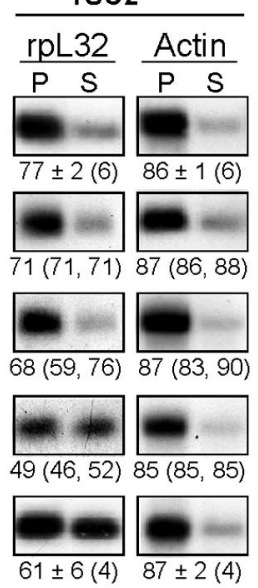

E
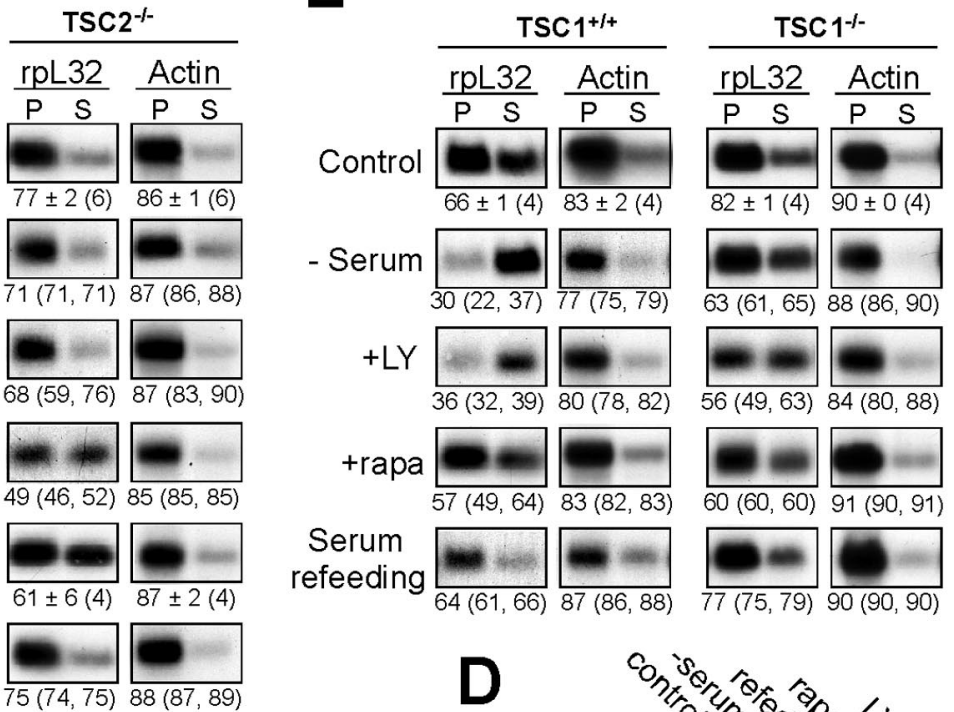

B

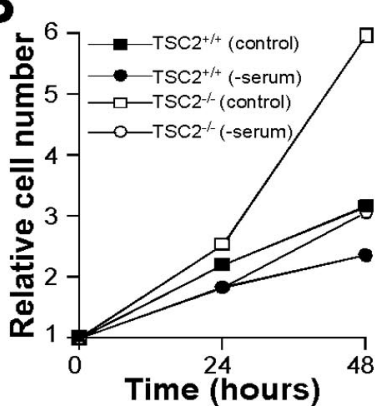

C

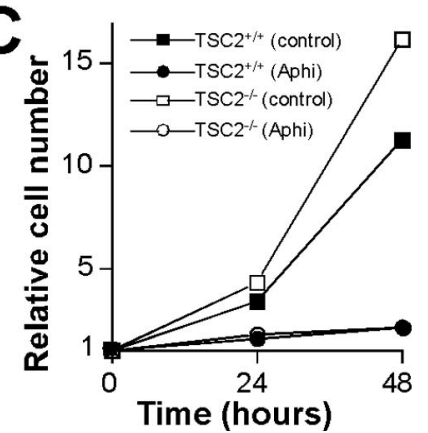

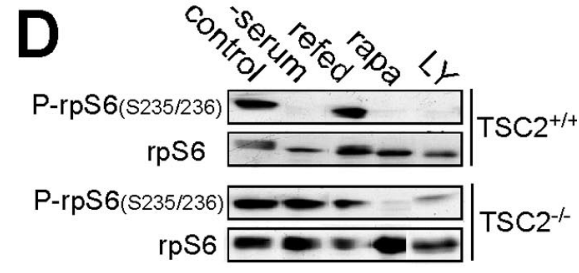

$\mathbf{F}$

P-rpS6(\$235/236) rpS6

FIG. 2. TSC2 or TSC1 deficiency rescues TOP mRNAs from translational repression in mitotically arrested cells. (A) TSC2 ${ }^{+/+}$and TSC2 ${ }^{-/-}$ MEFs were untreated (control), serum starved for $72 \mathrm{~h}$ (-serum), treated with aphidicolin for $48 \mathrm{~h}(+\mathrm{Aphi}), \mathrm{LY} 294002 \mathrm{for} 0.5 \mathrm{~h}(+\mathrm{LY})$, or rapamycin (+Rapa) for $2 \mathrm{~h}$, or were serum starved and then refed for $3 \mathrm{~h}$. Cells were harvested and subjected to polysomal analysis. (B) TSC2 ${ }^{+/+}$ and TSC2 $2^{-1-}$ cells were seeded in 96-well plates at a density of $4 \times 10^{3}$ cells per well. Cells were either untreated (control) or serum starved ( - serum) for the indicated amount of time. Proliferation was monitored by the methylene blue staining protocol (39). Absorbance measured $24 \mathrm{~h}$ after platting, set arbitrarily at 1 , and measured at later time points (average \pm SEM $[n=12]$ for each time point) was normalized to that value. (C) $\mathrm{TSC}^{+/+}$and $\mathrm{TSC}^{-/-}$cells were seeded in 96-well plates at a density of $4 \times 10^{3}$ cells per well. Cells were either untreated (control) or aphidicolin-treated (Aphi) for the indicated amount of time. Proliferation was monitored and presented as described in panel B. (D) TSC2 ${ }^{+/+}$and $\mathrm{TSC}^{-1-}$ cells were untreated (control), serum starved for $72 \mathrm{~h}$ (-serum), serum refed for $3 \mathrm{~h}$ (refed), rapamycin treated for $2 \mathrm{~h}$ (rapa), or LY294002 treated for $0.5 \mathrm{~h}(\mathrm{LY})$, and then harvested. The cytoplasmic proteins were subjected to Western blot analysis using the indicated antibodies. (E and F) Experimental details are similar to those described in panels A and D, respectively, except for the usage of TSC1 ${ }^{+/+}$and $\mathrm{TSC}^{+/+}$MEFs.

phase by aphidicolin treatment (Fig. 2A). The apparent rescue of TOP mRNA translation in $\mathrm{TSC}^{-1-}$ cells cannot be ascribed to an acquired resistance of these cells to the mitotic arrest, as serum starvation and aphidicolin had a similar inhibitory effect on the proliferation of both $\mathrm{TSC}^{+/+}$and $\mathrm{TSC}^{-/-}$ MEFs (Fig. 2B and C).

Next, we hypothesized that if the PI3K-Akt pathway exerts its stimulatory effect on TOP mRNAs solely through inactivation of the TSC1-TSC2 complex, then this pathway might be dispensable for efficient translation of TOP mRNAs in TSC2 $^{-1-}$ MEFs. To address this possibility, we applied the PI3K inhibitor LY294002 to TSC2 ${ }^{-1-}$ MEFs. Figure 2A shows that LY294002 elicited a 2.2- and 1.6-fold decrease in the percentage of rpL32 mRNA loaded on polysomes in TSC $2^{+/+}$ and $\mathrm{TSC}^{-1-}$ MEFs, respectively. These results imply that even though the translation of TOP mRNAs is repressed to a lesser extent in the mutant MEFs, these cells are still sensitive to the drug. This may be due to the fact that mTOR, which resides downstream of the TSC1-TSC2 complex, is also a target of LY294002 (8). However, complete inhibition of mTORC1 by rapamycin treatment, as can be judged by the phosphorylation status of rpS6 (Fig. 2D), had only a minor repressive effect on the translation efficiency of rpL32 in $\mathrm{TSC}^{+/+}$and $\mathrm{TSC}^{-/-}$MEFs (by a factor of 1.2 and 1.3, respectively) (Fig. 2A). These results imply that (i) the LY294002-sensitive signal is not transduced to translational efficiency of TOP mRNAs solely through the TSC1-TSC2 complex and that (ii) mTORC1 has a minor role in translational regulation of TOP mRNAs in these cell lines.

It should be noted that TOP mRNAs displayed similar re- 


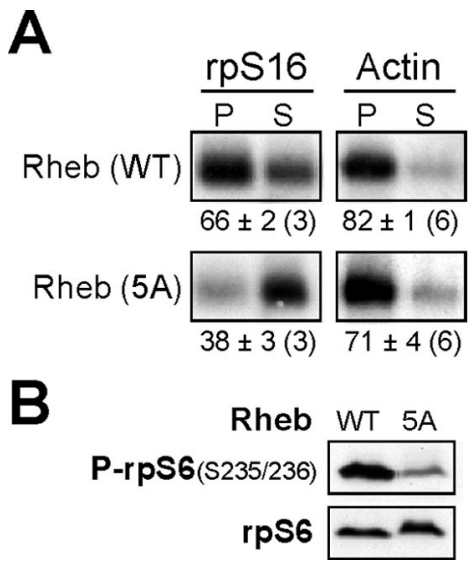

FIG. 3. Overexpression of Rheb can relieve translational repression of TOP mRNAs in mitotically arrested cells. (A) HEK 293 cells were transiently transfected with expression vectors encoding FLAGRheb (WT) or Myc-Rheb-5A, and $24 \mathrm{~h}$ later they were serum starved for $36 \mathrm{~h}$. Subsequently, cells were harvested and subjected to polysomal analysis. (B) HEK 293 cells were transiently transfected with expression vectors encoding FLAG-Rheb (WT) or Myc-Rheb (5A), and $24 \mathrm{~h}$ later they were serum starved for $36 \mathrm{~h}$. Subsequently, cells were harvested and cytoplasmic proteins were subjected to Western blot analysis using the indicated antibodies.

sistance to serum starvation in $\mathrm{TSC}^{-/-}$cells and $\mathrm{TSC}^{-/-}$ cells (Fig. 2E and F). Taken together, these results suggest that translational repression of TOP mRNAs upon quiescence relies on both TSC1 and TSC2. It is likely that the silencing of the TSC1-TSC2 complex constitutes a critical step toward translational activation of TOP mRNAs by mitotic stimuli.

Overexpression of Rheb can alleviate the translational repression of TOP mRNAs in quiescent cells. The TSC1-TSC2 complex acts as a GTPase activator toward Rheb by directly promoting the conversion of Rheb-GTP to Rheb-GDP (24, 53), thus inactivating Rheb. The inhibitory action of the TSC1TSC2 complex on Rheb is attenuated by Akt-catalyzed TSC2 phosphorylation (23). Hence, we assumed that overexpression of Rheb might bypass the inhibitory effect of the active TSC1TSC2 complex. To address this possibility, we measured the translation efficiency of rpS16 mRNA in HEK 293 cells transiently transfected with wild-type Rheb or Rheb defective in its effector domain (Rheb-5A) (24). Indeed, overexpression of wild-type Rheb, but not of Rheb-5A, elicited translation of TOP mRNAs in serum-starved cells (Fig. 3A) as well as protected rpS6 from dephosphorylation (Fig. 3B). These results suggest that TSC1-TSC2-dependent inactivation of Rheb mediates the downregulation of TOP mRNA translation.

mTOR deficiency represses TOP mRNA translation. The prevailing model of signaling from the TSC1-TSC2 complex through Rheb to mTOR (58) and conflicting reports on the translational repression of TOP mRNAs by rapamycin (see Table S1 in the supplemental material) prompted us to verify the role of mTOR in signaling toward these mRNAs. To address this question, we applied RNA interference in an attempt to knock down mTOR. Infection of HEK 293 cells with a lentivirus expressing mTOR shRNA blocked mTORC1 activity, as can be judged by the phosphorylation status of S6K upon insulin stimulation (Fig. 4A). Furthermore, mTOR knockdown

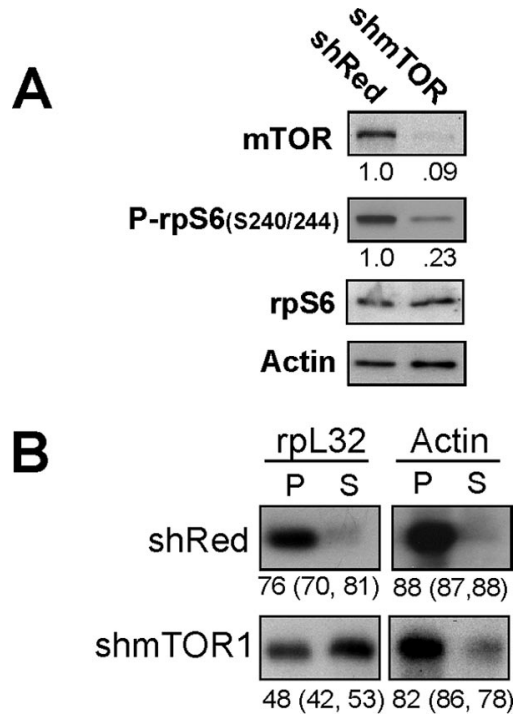

FIG. 4. Knockdown of mTOR downregulates the translation efficiency of TOP mRNAs in insulin-treated cells. (A) HEK 293 cells were infected with viruses expressing Red shRNA (shRed) or mTOR shRNA (shmTOR). Cells were 48-h serum starved and then insulin stimulated for $3 \mathrm{~h}$. The abundance of mTOR and its activity were monitored by Western blot analysis of cytoplasmic proteins with the indicated antibodies. The relative abundance of mTOR was normalized to that of actin, whereas the relative abundance of phospho rpS6 (P-rpS6) was normalized to that of rpS6. The results are numerically presented relative to those obtained for infected cells that expressed Red shRNA, which were arbitrarily set at 1. (B) Cytoplasmic extracts from cells described in panel A were subjected to polysomal analysis.

impaired the translation efficiency of TOP mRNAs in insulintreated cells (Fig. 4B). These results attest to the role of mTOR as a determinant of TOP mRNA translation.

The translation efficiency of TOP mRNAs is relatively resistant to raptor or rictor deficiency. The discrepancy between the apparent involvement of mTOR in translational control of TOP mRNAs and the relative resistance of the latter to rapamycin treatment, at least in some cell lines, prompted us to examine the role of raptor in the regulation of TOP mRNAs. To this end, we effectively knocked down raptor in HEK 293 cells, as could be judged by the decrease in its abundance and mTORC1 activity (Fig. 5A). Interestingly, raptor deficiency failed to downregulate the translation efficiency of rpL32 mRNAs in insulin-treated cells (Fig. 5B). This result suggests that either raptor is not essential for efficient translation of TOP mRNAs or, alternatively, the residual raptor expression $(\sim 10 \%)$ in each cell is sufficient for translational activation of these mRNAs. To distinguish between these two possibilities, we used mouse MEFs whose raptor gene could be conditionally knocked out by 4-hydroxytamoxifen (tamoxifen)-inducible Cre recombinase (iRapKO). Indeed, the apparent decrease in raptor upon tamoxifen treatment reflects a loss of raptor gene in $90 \%$ of the cells rather than a comparable loss of raptor in each cell (Fig. 5C). Nevertheless, most (68\%) of the rpL32 mRNA in tamoxifen-treated iRapKO cells was still recruited into polysomes (Fig. 5D). Moreover, the minor decrease in the translation efficiency of L32 mRNA can be partially attributed to the apparent diminution in mTOR abundance (by $30 \%$ to $50 \%$ ) that accompanied raptor knockout (Fig. 5C and data not 


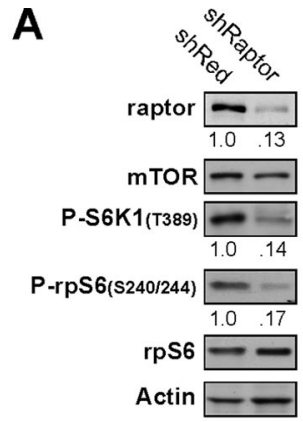

B

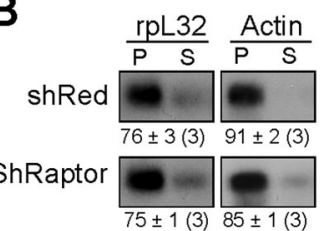

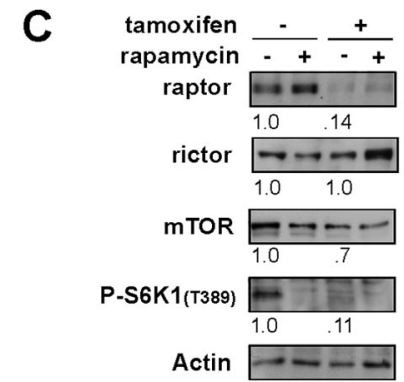

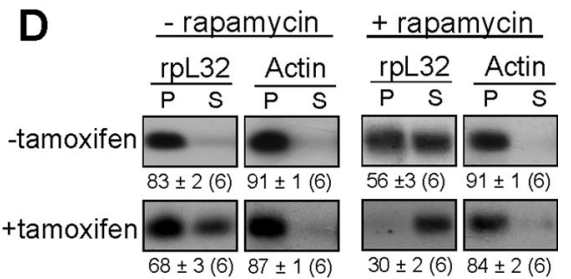

FIG. 5. The translation efficiency of TOP mRNAs does not rely on insulin-treated cells. (A) HEK 293 cells were infected with viruses expressing Red shRNA (shRed) or raptor shRNA (shRaptor). Cells were treated as described in the legend to Fig. 4. The abundance of raptor- and mTOR-dependent proteins was monitored by Western blot analysis with the indicated antibodies. The relative abundance of raptor, phospho S6K1(Thr389) and phospho Akt(Ser473) was normalized to that of actin, whereas the relative abundance of phospho rpS6(Ser235/236) was normalized to that of rpS6. The results are numerically presented relative to those obtained with Red shRNA-expressing cells that were arbitrarily set at 1. (B) Cytoplasmic extracts from cells described in panel A were subjected to polysomal analysis. (C) iRapKO cells were either untreated $(-)$ or treated $(+)$ with tamoxifen for 4 days and were then further incubated for $3 \mathrm{~h}$ in the absence $(-)$ or presence $(+)$ of rapamycin. The relative abundance of the indicated proteins was assessed as described in panel A with the exception that results are numerically presented relative to those obtained with cells untreated with either tamoxifen or rapamycin. (D) iRapKO cells were either treated or untreated with tamoxifen (for 4 days) and subsequently were harvested and subjected to polysomal analysis.

shown). It appears, therefore, that the translation efficiency of TOP mRNAs is nearly refractory to raptor deficiency.

In light of these results, we examined the seemingly default possibility, namely, that mTOR exerts its positive regulatory effect on TOP mRNAs via mTORC2. To this end, we took advantage of inducible rictor knockout MEFs, iRicKO. Surprisingly, the translation of TOP mRNAs was activated upon insulin treatment (Fig. 6A), even though more than $90 \%$ of the cells lost rictor expression (Fig. 6B) upon tamoxifen treatment. Taken together, our results suggest that either raptor or rictor is almost completely dispensable for efficient translation of TOP mRNAs.

Inhibition of TOP mRNA translation by rapamycin is sporadic, FKBP12 dependent, and raptor sensitive and does not involve mitotic arrest. TOP mRNAs display diverse responses to rapamycin treatment. This is exemplified by the relative resistance shown earlier (Fig. 1 and 2) on the one hand and the considerable repression of rpL32 mRNA in 3T3-L1 preadipocytes (Fig. 7A) on the other hand. This inconsistency, unlike the invariable inhibitory effect of rapamycin on S6K activity or rpS6 phosphorylation, posed a question regarding the mechanism involved in the rapamycin-dependent translational inhibition of TOP mRNAs. First, we examined whether FKBP12, which is required for inhibition of mTOR by rapamycin, is also involved in the translational repression of TOP mRNAs. Indeed, FK506, a small molecule that competes with rapamycin for binding to FKBP12, relieved both mTORC1 inhibition, as monitored by phosphorylation of S6K1 and rpS6 (Fig. 7B), and translational repression of TOP mRNAs (Fig. 7A). These results suggest that rapamycin exerts its inhibitory effect on TOP mRNAs in an FKBP12-dependent manner.

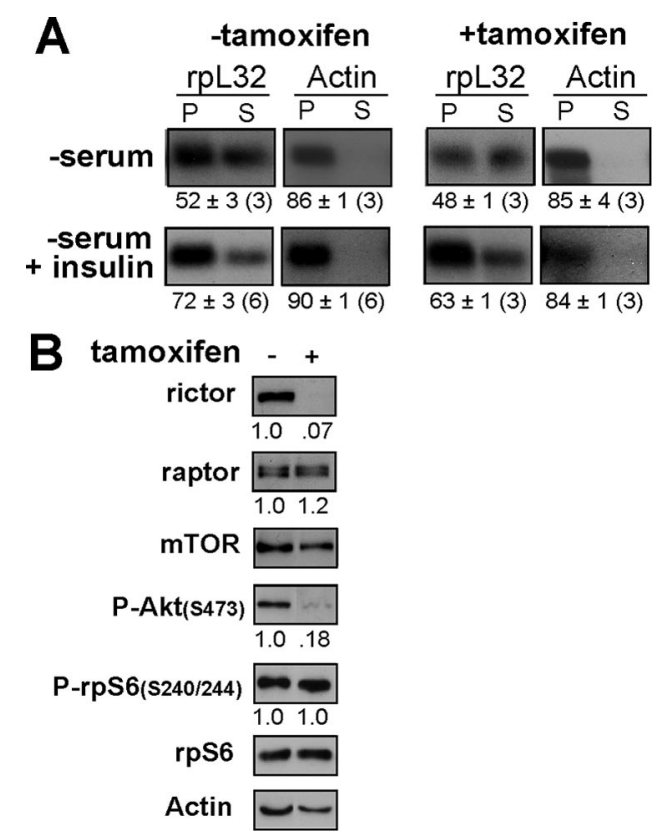

FIG. 6. TOP mRNAs are translationally activated by insulin in a rictor-independent fashion. (A) iRicKO cells were either untreated $(-)$ or treated $(+)$ with $1 \mu \mathrm{M}$ tamoxifen for 4 days and then serum starved for the last $48 \mathrm{~h}$ followed by 3 -h insulin treatment. The relative abundance of the indicated proteins was assessed as described in the legend to Fig. 5A. (B) iRicKO cells were either untreated $(-)$ or treated $(+)$ with $1 \mu \mathrm{M}$ tamoxifen for 4 days and then serum starved for the last $48 \mathrm{~h}$ followed by $3 \mathrm{~h}$ without $(-$ serum $)$ or with $(-$ serum + insulin) insulin treatment. Subsequently, cells were harvested and subjected to polysomal analysis. 


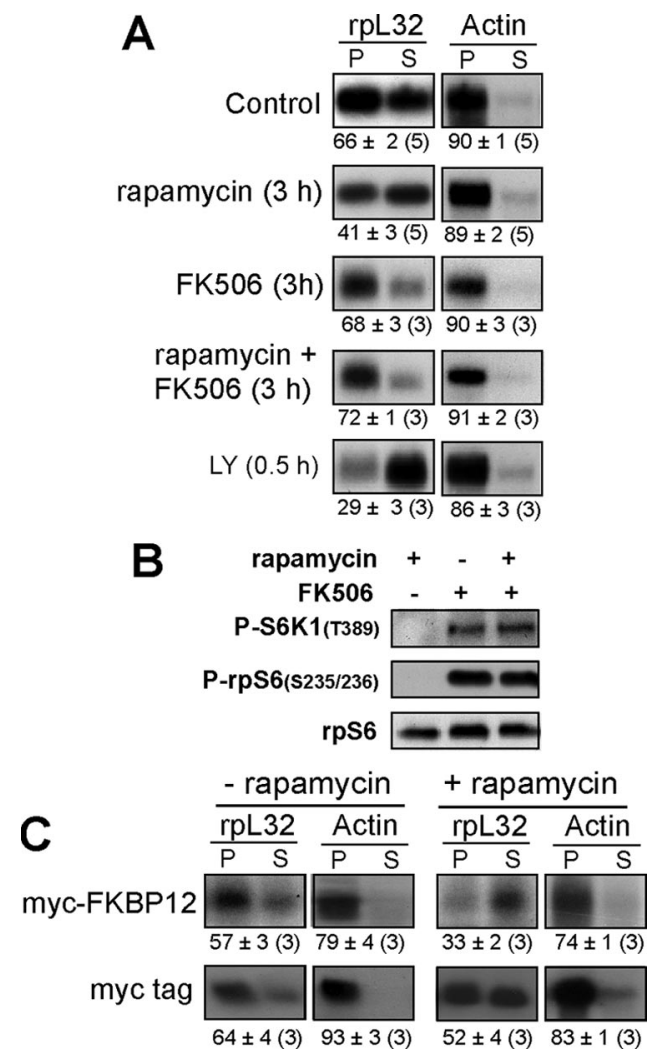

FIG. 7. Rapamycin represses the translation of TOP mRNAs in an FKBP-12-dependent and raptor-sensitive fashion. (A) 3T3-L1 preadipocytes were either untreated (Control), treated for $3 \mathrm{~h}$ with rapamycin, FK506, or both drugs, or treated for $0.5 \mathrm{~h}$ with LY294002. Subsequently, they were harvested and subjected to polysomal analysis. (B) 3T3-L1 preadipocytes were treated for $3 \mathrm{~h}$ with rapamycin, FK506, or both drugs. The cytoplasmic proteins were subjected to Western blot analysis. (C) HEK 293 cells were transiently transfected with pcDNA3-6myc-hFKBP12 (myc-FKBP12) or pcDNA3-6myc (myc tag). Forty-eight hours later, cells were incubated without $(-)$ or with $(+)$ rapamycin and were subsequently harvested and subjected to polysomal analysis.

TOP mRNA translation was markedly inhibited by rapamycin in raptor-deficient MEFs compared to the relative resistance observed in raptor-expressing cells (Fig. 5D). Notably, this rapamycin hypersensitivity was not observed with iRicKO cells (I. Patursky-Polischuk and O. Meyuhas, unpublished data), implying that it is not the tamoxifen treatment that sensitized the cells. It appears, therefore, that raptor not only does not mediate the inhibition of TOP mRNAs translation by rapamycin, it provides protection against this inhibition in the examined cells. Conceivably, the depletion of mTORC1 renders a novel mTOR activity (possibly free mTOR or mTORC3) that regulates TOP mRNA translation more accessible to the FKBP12-rapamycin complex. We assumed, therefore, that high levels of mTORC1 might be able to efficiently sequester much of the FKBP12-rapamycin complex, thus protecting mTOR-dependent TOP mRNA translation. If this is indeed the case, then rapamycin would more efficiently repress the translation of TOP mRNAs upon reduction of the mTORC1/FKBP12 ratio, as in the case of raptor knockout, or alternatively upon overproduction of FKBP12. Indeed,
FKBP12 overexpression in HEK 293 rendered the translation of TOP mRNAs rapamycin hypersensitive, which otherwise exhibited a relative rapamycin resistance (compare Fig. 7C and 1D).

The fact that the translation of TOP mRNAs is repressed upon mitotic arrest by any means (48) and that rapamycin can completely block progression through the cell cycle, at least in some cell types $(1,38,45)$, suggested a causal relationship between the effect of rapamycin on cell cycle progression and on TOP mRNAs. However, the lack of any correlation between the effects of rapamycin on the mitogenic activity of HEK 293 and 3T3-L1 cells (data not shown) on the one hand and on the translation efficiency of TOP mRNAs in these cell lines on the other hand (Fig. 1D and 7A) have disproved this notion. Taken together, our results indicate that the translational repression of TOP mRNAs by rapamycin relies on the availability of FKBP12, is greatly enhanced by depletion of raptor or overexpression of FKBP12, and is not mediated by mitotic arrest.

\section{DISCUSSION}

Insulin-induced translational activation of TOP mRNAs. Elucidating the pathway that transduces the insulin signal into translation activation of TOP mRNAs involved the following observations. First, insulin-induced relief of the translational repression of TOP mRNAs in serum-starved cells can be recapitulated by overexpression of constitutively active PI3K or Akt1 (49). Second, the PI3K inhibitor LY294002 rapidly and completely blocks the stimulatory effect of insulin (Fig. 1). Notably, this effect cannot be attributed to the documented ability of this drug to inhibit mTOR (8), as direct inhibition of mTOR by rapamycin failed to repress the translation of TOP mRNAs in the same cell lines (Fig. 1). Nevertheless, we cannot exclude the possibility that LY294002 exerts its inhibitory effect on TOP mRNAs via one of its newly identified (4) or as-yet-unidentified targets. Third, it has previously been shown that insulin stimulation of Akt leads to phosphorylation and inactivation of TSC2 $(12,23)$ and consequently to derepression of Rheb (14). Indeed, data presented here show that the stimulatory effect of insulin on translation efficiency of TOP mRNAs can be mimicked by knockout of TSC1 or TSC 2 or by Rheb overexpression. Interestingly, a similar rescue of TOP mRNA translation has been observed by microarray analysis of RNAs from serum-starved $\mathrm{TSC}^{-1-}$ or $\mathrm{TSC}^{-1-}$ cells (6). Fourth, the involvement of mTOR as a determinant of translation efficiency of TOP mRNAs is based primarily on the ability of mTOR knockdown to suppress the translational activation of these mRNAs by insulin (Fig. 4) or amino acids (data not shown).

If indeed the sole role of the PI3K-Akt domain of the pathway is to inactivate the TSC1-TSC2 complex, then translation of TOP mRNAs should be refractory to inhibition of PI3K in cells deficient for TSC1 or TSC2. However, our results suggest that deficiency of either of these proteins only partially relieved the LY294002-dependent translational repression of TOP mRNAs (Fig. 2). This latter observation might reflect signaling of PI3K, or another LY294002 target, to TOP mRNAs through an additional route.

Taken together, our results are consistent with a model 
where insulin induces PI3K and its target Akt, which in turn inactivates the TSC1-TSC2 complex; consequently, Rheb and its immediate target $\mathrm{mTOR}$ are derepressed, leading to translational activation of TOP mRNAs. It appears, therefore, that insulin induces protein synthesis by combining short- and longterm mechanisms. The first involves activation of translational initiation and elongation factors (reviewed in reference 43), whereas the second relies on the augmented capacity of protein synthesis by increasing the amount of the protein synthesis machinery through activation of TOP mRNAs translation, as well as transcription of ribosomal DNA (the present report and reference 2).

mTOR and TOP mRNAs. mTOR has been implicated in regulation of protein synthesis through multiple effectors (15, $19,44)$. However, even though mTOR has been referred to as a master regulator of protein synthesis (52), its blockage elicited 20 to $25 \%$ inhibition of global protein synthesis in cells treated with rapamycin for up to $4 \mathrm{~h}(25,33,41,55,57)$ or twice as much if treated for 20 to $24 \mathrm{~h}(5,54)$. These observations have led to the assumption that it is only a subset of mRNAs that are translationally repressed when mTOR is inhibited by rapamycin (see reference 16 and references therein). One class of mRNAs, whose translation has been implicated in this mode of regulation, through inactivation of $\mathrm{S} 6 \mathrm{~K}$ and consequently dephosphorylation of rpS6, is TOP mRNAs $(25,54)$. However, later studies with cells from $\mathrm{S} 6 \mathrm{~K} 1^{-1-} / \mathrm{S} 6 \mathrm{~K} 2^{-1-}$ double knockout mice and knock-in mice, carrying mutations in all phosphorylation sites of rpS6, have disproved any role of S6K activation or rpS6 phosphorylation in translational control of TOP mRNAs $(42,45)$. Nevertheless, the mTOR knockdown experiment presented here (Fig. 4) provides the first direct evidence for the involvement of mTOR as a positive regulator of TOP mRNA translation. Moreover, in a parallel study, we have shown that the translational efficiency of TOP mRNAs is rescued in cells that were amino acid activated in the presence of rapamycin if they expressed rapamycin-resistant mTOR mutant, but not if they expressed a catalytically inactive version of this mutant (Patursky-Polischuk and Meyuhas, unpublished). These results clearly attest to the positive regulatory role of mTOR catalytic activity in translation efficiency of TOP mRNAs and exclude the possibility that the results obtained with mTOR shRNA reflect an off-target effect. mTOR, being also a transducer of insulin and amino acid signals to rRNA synthesis (see reference 32 and references therein), appears to be a critical regulator of ribosome synthesis.

Several lines of evidence contradict the possibility that mTOR operates within mTORC1 to control the translation efficiency of TOP mRNAs. (i) Rapamycin ubiquitously and completely inhibits mTORC1 activity, yet it fails to repress the translation of TOP mRNAs or has just a minor inhibitory effect in most experiments conducted thus far (see Table S1 in the supplemental material). (ii) The translation of TOP mRNAs is refractory to raptor knockdown in human cells or repressed only slightly in knockout mouse cells, even though mTORC1 activity toward S6K1 activity is markedly reduced in both cases (Fig. 5). This resistance to raptor deficiency is further highlighted by the marked repression observed upon knockdown of mTOR (Fig. 4). Hence, the minor decrease in translational efficiency in raptor knockout cells can be ascribed, at least partly, to the concomitant reduction in mTOR abundance (Fig.
5C and data not shown). (iii) TOP mRNA translation is rendered rapamycin hypersensitive in raptor-deficient cells. (iv) The kinetics of inhibition of TOP mRNA translation upon amino acid deprivation of HEK 293 cells do not parallel the kinetics of mTORC1 inhibition, as the latter is markedly faster (within $1 \mathrm{~h}$ ) and more effective than rapamycin-induced repression of TOP mRNA translation (50). The delayed response to rapamycin is underscored by the rapidly detectable inhibition of TOP mRNA translation (within $0.5 \mathrm{~h}$ ) by LY294002 (Fig. 1B, 2A and B, and 7A). It should be pointed out, however, that the delayed response to rapamycin might reflect the accumulation/disappearance of a secondary effector, rather than the activation/inactivation of a direct effector.

Showing that mTORC1 has a minor, if any, role in the translational control of TOP mRNAs has invoked the possibility that TOP mRNAs are controlled primarily by mTORC2. Conceptually, this possibility seemed consistent with the sporadic sensitivity to rapamycin of both TOP mRNA translation (see Table S1 in the supplemental material) and TORC2 activity, as well as the delayed response of these two readouts to rapamycin relative to the rapamycin sensitivity of mTORC1 activity (46). Nevertheless, our results (Fig. 6) have refuted a requirement for $\mathrm{mTORC} 2$ in translational activation of TOP mRNAs by insulin.

Data presented in this report suggest that mTOR can exert its effect on TOP mRNAs in a way that is not strictly dependent on its association with either raptor or rictor. Several explanations can be proposed for reconciling these observations with the canonical two-mTOR-complex model. (i) mTOR controls TOP mRNA translation through a third, asyet-unidentified complex (possibly mTORC3), and (ii) mTOR can control TOP mRNAs in a complex-independent fashion. According to either of these explanations, neither raptor nor rictor is critical for TOP mRNA regulation. (iii) Formally, we cannot exclude the possibility that in the absence of mTORC1, the translational regulation of TOP mRNAs is mediated by mTORC2 and vice versa. Examining this last explanation would have to wait until we establish conditional rictor and raptor double knockout MEFs. Clearly, all these unresolved issues, as well as the identity of the effector(s) of mTOR that selectively determines the translation efficiency of TOP mRNAs, will be the focus of our future studies.

\section{ACKNOWLEDGMENTS}

This work was supported by grants to O.M. from the United StatesIsrael Binational Science Foundation (BSF 2005034), the Israel Science Foundation (grant no. 296/05), the German-Israeli Foundation (grant no. 819/05f), and the Otto Stieber Foundation. M.N.H. acknowledges support from the Swiss National Science Foundation and the Canton of Basel.

We thank Howard Green for the 3T3-L1 cells, Derek LeRoith for the NIH-IR cells, David Kwiatkowski for the TSC2 $2^{+/} /{\mathrm{p} 53^{-/-}}$, TSC $2^{-/-} /$ $\mathrm{p} 53^{-/-}, \mathrm{TSCl}^{+/+}$, and $\mathrm{TSC1}^{-/-} \mathrm{MEF}$ lines (26), John Blenis for the FLAG-Rheb plasmid, Kum-Liang Guan for the Myc-Rheb(5A) plasmid, Akira Kurisaki for the pcDNA3-6myc-hFKBP12 plasmid, and Michal Gropp for the Sin18 plasmid.

\section{REFERENCES}

1. Abraham, R. T., and G. J. Wiederrecht. 1996. Immunopharmacology of rapamycin. Annu. Rev. Immunol. 14:483-510.

2. Antonetti, D. A., S. R. Kimball, R. L. Horetsky, and L. S. Jefferson. 1993 Regulation of rDNA transcription by insulin in primary cultures of rat hepatocytes. J. Biol. Chem. 268:25277-25284. 
3. Bai, X., D. Ma, A. Liu, X. Shen, Q. J. Wang, Y. Liu, and Y. Jiang. 2007. Rheb activates mTOR by antagonizing its endogenous inhibitor, FKBP38. Science 318:977-980.

4. Bain, J., L. Plater, M. Elliott, N. Shpiro, C. J. Hastie, H. McLauchlan, I. Klevernic, J. S. Arthur, D. R. Alessi, and P. Cohen. 2007. The selectivity of protein kinase inhibitors: a further update. Biochem. J. 408:297-315.

5. Beretta, L., A. Gingras, Y. Svitkin, M. Hall, and N. Sonenberg. 1996. Rapamycin blocks the phosphorylation of 4E-BP1 and inhibits cap-dependent initiation of translation. EMBO J. 15:658-664

6. Bilanges, B., R. Argonza-Barrett, M. Kolesnichenko, C. Skinner, M. Nair, M. Chen, and D. Stokoe. 2007. Tuberous sclerosis complex proteins 1 and 2 control serum-dependent translation in a TOP-dependent and -independent manner. Mol. Cell. Biol. 27:5746-5764.

7. Brummelkamp, T., R. Bernards, and R. Agami. 2002. A system for stable expression of short interfering RNAs in mammalian cells. Science 296:550553.

8. Brunn, G. J., J. Williams, C. Sabers, G. Wiederrecht, J. C. J. Lawrence, and R. T. Abraham. 1996. Direct inhibition of the signaling functions of the mammalian target of rapamycin by the phosphoinositide 3-kinase inhibitors, wortmannin and LY294002. EMBO. J. 15:5256-5267.

9. Cai, S. L., A. R. Tee, J. D. Short, J. M. Bergeron, J. Kim, J. Shen, R. Guo, C. L. Johnson, K. Kiguchi, and C. L. Walker. 2006. Activity of TSC2 is inhibited by AKT-mediated phosphorylation and membrane partitioning. J. Cell Biol. 173:279-289.

10. Chen, J., X. F. Zheng, E. J. Brown, and S. L. Schreiber. 1995. Identification of an 11-kDa FKBP12-rapamycin-binding domain within the 289-kDa FKBP12-rapamycin-associated protein and characterization of a critical serine residue. Proc. Natl. Acad. Sci. USA 92:4947-4951.

11. Chung, S., and R. P. Perry. 1989. Importance of introns for expression of mouse ribosomal protein gene rpL32. Mol. Cell. Biol. 9:2075-2082.

12. Dan, H. C., M. Sun, L. Yang, R. I. Feldman, X. M. Sui, C. C. Ou, M. Nellist, R. S. Yeung, D. J. Halley, S. V. Nicosia, W. J. Pledger, and J. Q. Cheng. 2002 Phosphatidylinositol 3-kinase/Akt pathway regulates tuberous sclerosis tumor suppressor complex by phosphorylation of tuberin. J. Biol. Chem. 277 35364-35370.

13. DePhilip, R. M., D. E. Chadwick, R. A. Ignotz, W. E. Lynch, and I. Lieberman. 1979. Rapid stimulation of ribosome synthesis in cultured chick embryo fibroblasts. Biochemistry 18:4812-4817.

14. Garami, A., F. J. Zwartkruis, T. Nobukuni, M. Joaquin, M. Roccio, H. Stocker, S. C. Kozma, E. B. Hafen, J. L. Bos, and G. Thomas. 2003. Insulin activation of Rheb, a mediator of mTOR/S6K/4E-BP signaling, is inhibited by TSC1 and 2. Mol. Cell 11:1457-1466.

15. Gingras, A. C., S. P. Gygi, B. Raught, R. D. Polakiewicz, R. T. Abraham, M. F. Hoekstra, R. Aebersold, and N. Sonenberg. 1999. Regulation of 4EBP1 phosphorylation: a novel two-step mechanism. Genes Dev. 13:14221437.

16. Gingras, A. C., B. Raught, and N. Sonenberg. 2001. Regulation of translation initiation by FRAP/mTOR. Genes Dev. 15:807-826.

17. Green, H., and O. Kehinde. 1974. Sublines of mouse 3 T3 cells that accumulate lipid. Cell 1:113-116.

18. Gropp, M., P. Itsykson, O. Singer, T. Ben-Hur, E. Reinhartz, E. Galun, and B. E. Reubinoff. 2003. Stable genetic modification of human embryonic stem cells by lentiviral vectors. Mol. Ther. 7:281-287.

19. Harris, T. E., A. Chi, J. Shabanowitz, D. F. Hunt, R. E. Rhoads, and J. C. J. Lawrence. 2006. mTOR-dependent stimulation of the association of eIF4G and eIF3 by insulin. EMBO J. 25:1659-1668.

20. Hornstein, E., H. Harel, G. Levy, and O. Meyuhas. 1999. Overexpression of poly(A)-binding protein down-regulates the translation or the abundance of its own mRNA. FEBS Lett. 457:209-213.

21. Hresko, R. C., and M. Mueckler. 2005. mTOR.RICTOR is the Ser473 kinase for Akt/protein kinase B in 3T3-L1 adipocytes. J. Biol. Chem. 280:4040640416

22. Inoki, K., M. N. Corradetti, and K. L. Guan. 2005. Dysregulation of the TSC-mTOR pathway in human disease. Nat. Genet. 37:19-24.

23. Inoki, K., L. Li, J. Zhu, J. Wu, and K. L. Guan. 2002. TSC2 is phosphorylated and inhibited by Akt and suppresses mTOR signalling. Nat. Cell Biol. 4:648 657.

24. Inoki, K., Y. Li, T. Xu, and K. L. Guan. 2003. Rheb GTPase is a direct target of TSC2 GAP activity and regulates mTOR signaling. Genes Dev. 17:18291834.

25. Jefferies, H. B. J., C. Reinhard, S. C. Kozma, and G. Thomas. 1994. Rapamycin selectively represses translation of the 'polypyrimidine tract' mRNA family. Proc. Natl. Acad. Sci. USA 91:4441-4445.

26. Kwiatkowski, D. J., H. Zhang, J. L. Bandura, K. M. Heiberger, M. Glogauer, N. el-Hashemite, and H. Onda. 2002. A mouse model of TSC1 reveals sex-dependent lethality from liver hemangiomas, and up-regulation of p70S6 kinase activity in Tsc1 null cells. Hum. Mol. Genet. 11:525-534.

27. Lalanne, J., M. Lucero, and J. le Moullec. 1987. Complete sequence of mouse S6 ribosomal protein. Nucleic Acids Res. 15:4990.

28. Levenson, R. M., A. C. Nairn, and P. J. Blackshear. 1989. Insulin rapidly induces the biosynthesis of elongation factor 2. J. Biol. Chem. 264:11904 11911.
29. Levy-Toledano, R., D. Accili, and S. I. Taylor. 1993. Deletion of C-termina 113 amino acids impairs processing and internalization of human insulin receptor: comparison of receptors expressed in $\mathrm{CHO}$ and NIH-3T3 cells. Biochim. Biophys. Acta 1220:1-14

30. Long, X., Y. Lin, S. Ortiz-Vega, K. Yonezawa, and J. Avruch. 2005. Rheb binds and regulates the mTOR kinase. Curr. Biol. 15:702-713.

31. Manning, B. D., and L. C. Cantley. 2007. AKT/PKB signaling: navigating downstream. Cell 129:1261-1274.

32. Mayer, C., J. Zhao, X. Yuan, and I. Grummt. 2004. mTOR-dependent activation of the transcription factor TIF-IA links rRNA synthesis to nutrient availability. Genes Dev. 18:423-434.

33. Mèndez, R., M. G. J. Myers, M. F. White, and R. E. Rhoads. 1996. Stimulation of protein synthesis, eukaryotic translation initiation factor 4E phosphorylation, and PHAS-I phosphorylation by insulin requires insulin receptor substrate 1 and phosphatidylinositol 3-kinase. Mol. Cell. Biol. 16:2857-2864.

34. Meyuhas, O. 2000. Synthesis of the translational apparatus is regulated at the translational level. Eur. J. Biochem. 267:6321-6330.

35. Meyuhas, O., and R. P. Perry. 1980. Construction and identification of cDNA clones for several mouse ribosomal proteins. Application for the study of r-protein gene expression. Gene 10:113-129.

36. Minty, A. J., M. Caravatti, B. Robert, A. Cohen, P. Daubas, A. Weydert, F. Gross, and M. E. Buckingham. 1981. Mouse actin messenger RNAs: construction and characterization of a recombinant plasmid molecule containing a complementary DNA transcript of mouse a-actin mRNA. J. Biol. Chem. 256:1008-1014.

37. Naldini, L., U. Blomer, F. H. Gage, D. Trono, and I. M. Verma. 1996. Efficient transfer, integration, and sustained long-term expression of the transgene in adult rat brains injected with a lentiviral vector. Proc. Natl. Acad. Sci. USA 93:11382-11388.

38. Ohanna, M., A. K. Sobering, T. Lapointe, L. Lorenzo, C. Praud, E. Petroulakis, N. Sonenberg, P. A. Kelly, A. Sotiropoulos, and M. Pende. 2005. Atrophy of S6K1 $1^{-/}$skeletal muscle cells reveals distinct mTOR effectors for cell cycle and size control. Nat. Cell Biol. 7:286-294.

39. Oliver, M. H., N. K. Harrison, J. E. Bishop, P. J. Cole, and G. J. Laurent. 1989. A rapid and convenient assay for counting cells cultured in microwell plates: application for assessment of growth factors. J. Cell Sci. 92:513-518.

40. Parrott, L. A., and D. J. Templeton. 1999. Osmotic stress inhibits p70/85 S6 kinase through activation of a protein phosphatase. J. Biol. Chem. 274: 24731-24736.

41. Pedersen, S., J. E. Celis, J. Nielsen, J. Christiansen, and F. C. Nielsen. 1997. Distinct repression of translation by wortmannin and rapamycin. Eur. J. Biochem. 247:449-456.

42. Pende, M., S. H. Um, V. Mieulet, M. Sticker, V. L. Goss, J. Mestan, M. Mueller, S. Fumagalli, S. C. Kozma, and G. Thomas. 2004. S6K $1^{-/-}$ $\mathrm{S} 6 \mathrm{~K} 2^{-/-}$mice exhibit perinatal lethality and rapamycin-sensitive $5^{\prime}$-terminal oligopyrimidine mRNA translation and reveal a mitogen-activated protein kinase-dependent S6 kinase pathway. Mol. Cell. Biol. 24:3112-3124.

43. Proud, C. G. 2006. Regulation of protein synthesis by insulin. Biochem. Soc. Trans. 34:213-216.

44. Ruvinsky, I., and O. Meyuhas. 2006. Ribosomal protein S6 phosphorylation: from protein synthesis to cell size. Trends Biochem. Sci. 31:342-348.

45. Ruvinsky, I., N. Sharon, T. Lerer, H. Cohen, M. Stolovich-Rain, T. Nir, Y. Dor, P. Zisman, and O. Meyuhas. 2005. Ribosomal protein S6 phosphorylation is a determinant of cell size and glucose homeostasis. Genes Dev. 19:2199-2211.

46. Sarbassov, D. D., S. M. Ali, S. Sengupta, J. H. Sheen, P. P. Hsu, A. F. Bagley, A. L. Markhard, and D. M. Sabatini. 2006. Prolonged rapamycin treatment inhibits mTORC2 assembly and Akt/PKB. Mol. Cell 22:159-168.

47. Sarbassov, D. D., D. A. Guertin, S. M. Ali, and D. M. Sabatini. 2005. Phosphorylation and regulation of Akt/PKB by the rictor-mTOR complex Science 307:1098-1101.

48. Stolovich, M., T. Lerer, Y. Bolkier, H. Cohen, and O. Meyuhas. 2005. Lithium can relieve translational repression of TOP mRNAs elicited by various blocks along the cell cycle in a glycogen synthase kinase-3- and S6-kinaseindependent manner. J. Biol. Chem. 280:5336-5342.

49. Stolovich, M., H. Tang, E. Hornstein, G. Levy, R. Cohen, S. S. Bae, M. J. Birnbaum, and O. Meyuhas. 2002. Transduction of growth or mitogenic signals into translational activation of TOP mRNAs is fully reliant on the PI3-kinase-mediated pathway, but requires neither S6K1 nor rpS6 phosphorylation. Mol. Cell. Biol. 22:8101-8113.

50. Tang, H., E. Hornstein, M. Stolovich, G. Levy, M. Livingstone, D. J. Templeton, J. Avruch, and O. Meyuhas. 2001. Amino acid-induced translation of TOP mRNAs is fully dependent on phosphatidylinositol 3-kinase-mediated signaling, is partially inhibited by rapamycin, and is independent of S6K1 and rpS6 phosphorylation. Mol. Cell. Biol. 21:8671-8683.

51. Taniguchi, C. M., B. Emanuelli, and R. C. Kahn. 2006. Critical nodes in signalling pathways: insights into insulin action. Nat. Rev. Mol. Cell Biol. 7:85-96

52. Tee, A. R., and J. Blenis. 2005. mTOR, translational control and human disease. Semin. Cell Dev. Biol. 16:29-37.

53. Tee, A. R., B. D. Manning, P. P. Roux, L. C. Cantley, and J. Blenis. 2003. Tuberous sclerosis complex gene products, Tuberin and Hamartin, control 
mTOR signaling by acting as a GTPase-activating protein complex toward Rheb. Curr. Biol. 13:1259-1268.

54. Terada, N., H. R. Patel, K. Takase, K. Kohno, A. C. Nairn, and E. W. Gelfand. 1994. Rapamycin selectively inhibits translation of mRNAs encoding elongation factors and ribosomal proteins. Proc. Natl. Acad. Sci. USA 91:11477-11481.

55. Terada, N., K. Takase, P. Papst, A. C. Nairn, and E. W. Gelfand. 1995 Rapamycin inhibits ribosomal protein synthesis and induces $\mathrm{G} 1$ prolongation in mitogen-activated T lymphocytes. J. Immunol. 155:3418-3426.

56. Thedieck, K., P. Polak, M. L. Kim, K. D. Molle, A. Cohen, P. Jeno, C. Arrieumerlou, and M. N. Hall. 2007. PRAS40 and PRR5-like protein are new mTOR interactors that regulate apoptosis. PLoS One 2:e1217.

57. Wang, L., X. Wang, and C. G. Proud. 2000. Activation of mRNA translation in rat cardiac myocytes by insulin involves multiple rapamycin-sensitive steps. Am. J. Physiol. Heart Circ. Physiol. 278:H1056-H1068.

58. Wullschleger, S., R. Loewith, and M. N. Hall. 2006. TOR signaling in growth and metabolism. Cell 124:471-484.

59. Yang, Q., and K. L. Guan. 2007. Expanding mTOR signaling. Cell Res. 17:666-681.

60. Zhang, H., G. Cicchetti, H. Onda, H. B. Koon, K. Asrican, N. Bajraszewski, F. Vazquez, C. L. Carpenter, and D. J. Kwiatkowski. 2003. Loss of Tsc1/Tsc2 activates mTOR and disrupts PI3K-Akt signaling through downregulation of PDGFR. J. Clin. Investig. 112:1223-1233.

61. Zufferey, R., D. Nagy, R. J. Mandel, L. Naldini, and D. Trono. 1997. Multiply attenuated lentiviral vector achieves efficient gene delivery in vivo. Nat. Biotechnol. 15:871-875. 